\title{
PQMon: a powerful veto for burst events
}

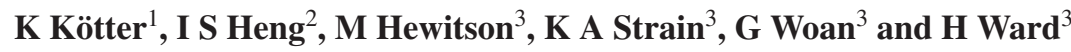 \\ ${ }^{1}$ Institut für Atom- und Molekülphysik, Universität Hannover, Callinstr. 38, 30167 Hannover, \\ Germany \\ 2 Max-Planck-Institut für Gravitationsphysik, Albert-Einstein-Institut, Hannover Callinstr. 38, \\ 30167 Hannover, Germany \\ ${ }^{3}$ Department of Physics \& Astronomy, University of Glasgow, Glasgow G12 8QQ, UK \\ E-mail: Karsten.Koetter@aei.mpg.de
}

Received 2 April 2003, in final form 9 June 2003

Published 18 August 2003

Online at stacks.iop.org/CQG/20/S895

\begin{abstract}
Data taken by the gravitational wave detector GEO 600 (Danzmann K et al 1994 GEO 600-Proposal for a 600m Laser-Interferometric Gravitational Wave Antenna (Garching: MPQ)) during the science run 'S1' contain a large number of transients. In order to reduce the false-alarm rate of burst gravitational wave search algorithms it is desirable to veto all those transients that are clearly not of gravitational wave origin. This paper presents a method of vetoing transients by looking at the 'in-phase' $(P)$ and 'quadrature' $(Q)$ channels of the demodulated interferometer output signal. By taking advantage of the fact that the $P$-channel and the $Q$-channel have different sensitivities to gravitational wave burst events, this method can veto transients that have a power distribution different from the signature of a signal induced by a gravitational wave.
\end{abstract}

PACS numbers: $04.80 . \mathrm{Nn}, 95.55 . \mathrm{Ym}$

(Some figures in this article are in colour only in the electronic version)

\section{Introduction}

The gravitational wave detector GEO 600 [1] recorded 17 days of data during a data-taking run called the 'S1 run' from 23 August to 9 September 2002 that was carried out in conjunction with GEO's LSC [2] partners from LIGO [3].

The data recorded in the gravitational wave strain channel (also called $h$-channel) of GEO 600 are used as an input for search algorithms looking for gravitational wave burst events. These algorithms (e.g. ExcessPower [4] and TFCLUSTERS [5]) find a large number of candidate burst events because the $h$-channel contains many transients that are most likely caused by environmental disturbances or instrumental noise. Therefore it is desirable to develop veto strategies that will eliminate a large fraction of these spurious events. 


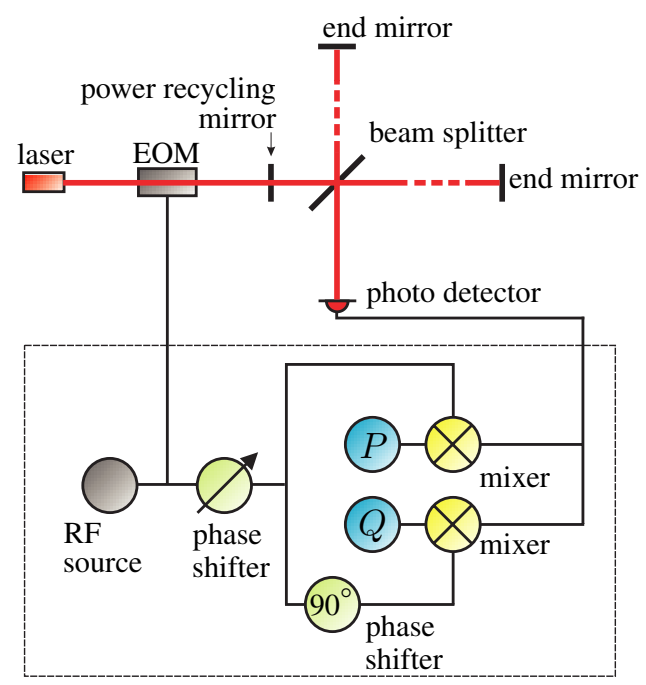

Figure 1. In-phase and quadrature channels of GEO 600.

While it is planned that environmental monitors will ultimately play a large part in the vetoing of spurious events, it is also appropriate to develop vetos that are based on differences of the interferometer responses to gravitational waves and to environmental triggers.

\section{Vetoing burst events using PQMon}

\subsection{Concept of the PQMon algorithm}

In the GEO 600 setup (see figure 1), the $h$-channel is generated by a method called 'frontal modulation'. The laser light that is injected into the interferometer is frequency modulated by an electro-optical modulator (EOM) driven by a $14.9 \mathrm{MHz}$ sine wave generator. By demodulating the signal coming from the output photo detector located at the output port of the interferometer, the in-phase $(P)$ and quadrature $(Q)$ channels are obtained.

A phase shifter is used to maximize the sensitivity to differential arm length changes of the $P$-channel. At the same time it minimizes the sensitivity of the $Q$-channel. This adjustment of the demodulation phase is never perfect (e.g. due to slow drifts that can be seen in figure 4) so that the sensitivity of the $Q$-channel is not zero but much lower than the sensitivity of the $P$-channel. The information contained in these channels allows identification of transients that are not caused by a differential arm length change in the interferometer and therefore cannot be of gravitational wave origin:

The relative sensitivity of the $P$ - and $Q$-channels to differential arm length change can be obtained by monitoring the power $P_{c}$ and $Q_{c}$ of the calibration lines in the respective channel. One can then look at a candidate burst event and determine the power $P_{b}$ and $Q_{b}$ in the two channels during the time of the event. The ratio $P_{b} / Q_{b}$ should be close to $P_{c} / Q_{c}$ for an event induced by a gravitational wave.

In summary the PQMon algorithm performs the following steps.

- Detects candidate burst event in the $h$-channel.

- Checks power distribution of candidate event in $P$ - and $Q$-channels $\left(P_{b} / Q_{b}\right)$. 


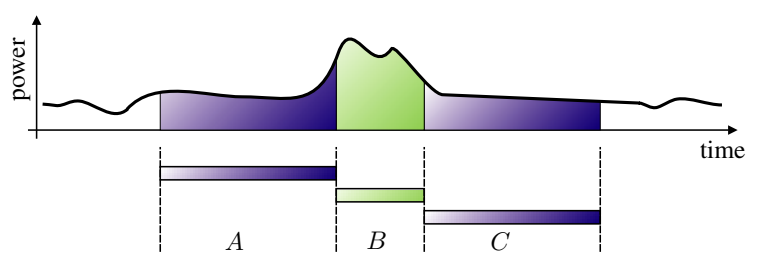

Figure 2. Time domain windows used for the transient detection algorithm.

- Determines relative sensitivity to differential arm length changes of $P$-channel and $Q$ channel $\left(P_{c} / Q_{c}\right)$ by measuring the calibration signal.

- Compares $P_{b} / Q_{b}$ and $P_{c} / Q_{c}$ and decides whether to veto the event or not.

\subsection{Transient detection}

For detection of transients in the data, a very simple algorithm is employed. Three adjacent time domain windows are moved along the data (see figure 2).

In order to detect a transient in window $B$ the average power per sample (called $\bar{B}$ ) is computed for this window that has a length of ten samples ( $\widehat{=} 610 \mu \mathrm{s})$. To get an estimation of the noise that is present in the data, the average power per sample is also computed for the combination of windows $A$ and $C($ called $\bar{N})$. Each of these windows has a length of 45 samples ( $\widehat{=} 2.74 \mathrm{~ms}$ ). The value $\bar{B} / \bar{N}$ represents the signal-to-noise ratio (SNR) in power of the event in window $B$. When this SNR crosses a given threshold, the time at the centre of window $B$ is considered as the start of a transient. This value is recorded together with the maximum SNR and the time when the SNR drops below the threshold again which marks the end of the event.

\subsection{Measuring the power ratio $P_{b} / Q_{b}$ of a candidate burst event}

To obtain the power distribution in the $P$ - and $Q$-channels for the transient, the same time domain windows (see figure 2) as for the transient detection were used. The windows are positioned so that the time of the peak of the candidate event is in the centre of window $B$. As for the transient detection, the noise floor is estimated by averaging the power in windows $A$ and $C$. This value is assumed to be the mean power of the underlying noise, $\left\langle n^{2}\right\rangle$, in window $B$. The overall signal power $S_{\text {winB }}^{2}$ in window $B$ is obtained by measuring the overall power in each time domain bin, $V_{i}^{2}$, corrected for the noise contribution, $\left\langle n^{2}\right\rangle$, which gives the estimated signal power per time domain bin, $\hat{S}_{i}^{2}$ :

$$
\hat{S}_{i}^{2}=V_{i}^{2}-\left\langle n^{2}\right\rangle \text {. }
$$

The variance of this estimation is

$$
\operatorname{var}\left(\hat{S}_{i}^{2}\right)=4 \hat{S}_{i}^{2}\left\langle n^{2}\right\rangle+2\left\langle n^{2}\right\rangle^{2} .
$$

The overall signal power in window $B$ is the sum of the signal power in all of its time domain bins:

$$
\hat{S}^{2}{ }_{\text {winB }}=\sum_{i} \hat{S}_{i}^{2}
$$

Under the assumption that the measurements of $\hat{S}_{i}^{2}$ are uncorrelated the value of $\hat{S}^{2}$ winB has a variance of

$$
\operatorname{var}\left(\hat{S}^{2}{ }_{\mathrm{winB}}\right)=\sum_{i} \operatorname{var}\left(\hat{S}_{i}^{2}\right)
$$




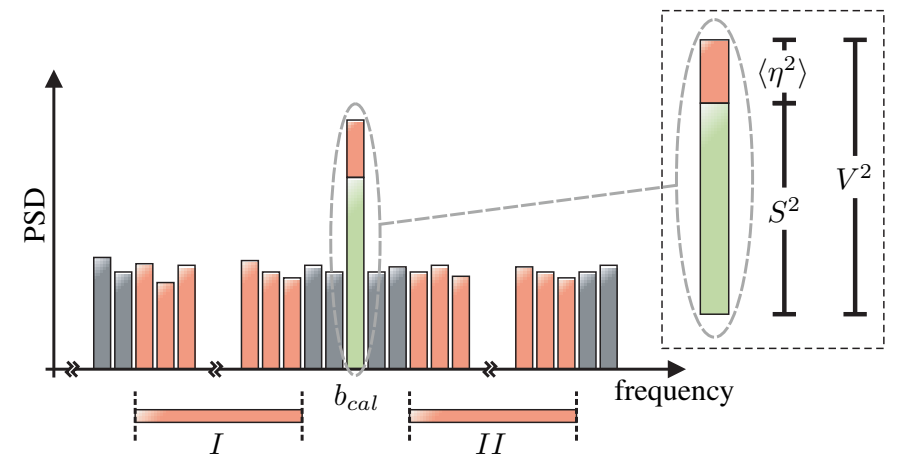

Figure 3. Measuring the noise floor and the calibration line signal: the frequency bin $b_{\text {cal }}$ contains the calibration line; windows $I$ and $I I$ are used to determine the noise floor.

Measuring the power and its variance in the central window in the channels $P$ and $Q$ gives the values for $P_{b}$ and $Q_{b}$ so that the ratio $P_{b} / Q_{b}$ and the variance of $P_{b} / Q_{b}$ can be determined.

\subsection{Measuring the calibration line ratio $P_{c} / Q_{c}$}

To calibrate the output signal of the GEO 600 interferometer, a set of calibration lines is continuously injected into the instrument. This is done by moving the end mirrors in a controlled way at three different frequencies [6]. By comparing the response of the $P$ - and $Q$ channels to the calibration signal, the relative sensitivity of these channels can be determined.

By locking the calibration frequencies to the sample clock of the data acquisition system, we ensure that the calibration lines are not drifting with respect to the sampling frequency. Therefore all power of a line will show up in a single frequency bin, $b_{\text {cal }}$, of a power spectral density (PSD) provided the frequency resolution is chosen such that the calibration line is in the centre of a frequency bin.

The overall power, $V^{2}$, measured in the calibration line frequency bin, $b_{\text {cal }}$, will be the sum of the power, $S^{2}$, originating from the calibration line and the power, $\eta^{2}$, due to noise.

To determine the contribution of the noise in $b_{\text {cal }}$, it is assumed that the noise can be modelled as Gaussian white noise.

This means that the variance of the power in a frequency bin can be determined from the average power in that bin by

$$
\operatorname{var}\left(\eta^{2}\right)=\left\langle\eta^{2}\right\rangle^{2} / N
$$

where $N$ is the number of averages used to compute the PSD.

Measurement and averaging of the noise power per bin, $\eta^{2}$, is illustrated in figure 3. By measuring frequency bins around $b_{\text {cal }}$ (bins in regions $I$ and $I I$ ) that do not contain power from the calibration signal, the average value, $\left\langle\eta^{2}\right\rangle$, can be determined.

From the measurement of the overall power, $V^{2}$, in the calibration frequency bin one can get an estimation of the calibration signal power, $\hat{S}^{2}$, by subtracting the average noise power, $\left\langle\eta^{2}\right\rangle$ :

$$
\hat{S}^{2}=V^{2}-\left\langle\eta^{2}\right\rangle \text {. }
$$

Using equation (5) it can be shown that the variance of $\hat{S}^{2}$ is given by

$$
\operatorname{var}\left(\hat{S}^{2}\right)=\left(2 \hat{S}^{2}\left\langle\eta^{2}\right\rangle+\left\langle\eta^{2}\right\rangle^{2}\right) / N
$$


The ratio $R_{\mathrm{cal}_{i}}$ for each calibration line is calculated by

$$
R_{\mathrm{cal}_{i}}=P_{c_{i}} / Q_{c_{i}}
$$

where $P_{c_{i}}$ and $Q_{c_{i}}$ are the measured powers of the $i$ th calibration line frequency in the $P$ - and $Q$-channel, respectively. The variance of the value $R_{\mathrm{cal}_{i}}$ is called $\sigma_{R_{\mathrm{cal}}{ }_{i}}$.

For the optical configuration of GEO 600 used during the S1 run the relative sensitivity to differential arm length change of the $P$ - and $Q$-channels is frequency independent. Therefore, the values $R_{\mathrm{cal}_{i}}$ for the three calibration lines are equivalent and the three individual measurements can easily be combined to an overall value with a smaller variance than that of each individual measurement. The combined value of $R_{\mathrm{cal}}=P_{c} / Q_{c}$ can be calculated by a weighted sum of the individual measurements,

$$
R_{\mathrm{cal}}=\frac{\sum_{i} R_{\mathrm{cal}_{i}} \sigma_{R_{\mathrm{cal}_{i}}^{-2}}}{\sum_{i} \sigma_{R_{\mathrm{cal}_{i}}^{-}}} .
$$

The resulting variance of this value is

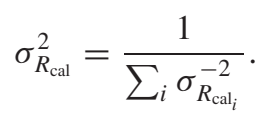

\section{Results}

The following graphs show results that were obtained from GEO 600 data recorded during the S1 run that took place from 15:00:00 23-08-2002 to 15:00:00 09-09-2002 (UTC). For more than $98 \%$ of the time, the detector produced valid data that could be used for analysis by PQMon.

The algorithm was implemented as a data analysis monitor written in GEO++ [7] which is a $\mathrm{C}++$ class library designed to process interferometer data. The monitor was run on data from the $P$ - and $Q$-channels. Before the algorithm was applied, the data were prewhitened to equalize the amount of power in different frequency bands. For the transient detection algorithm, the whitened $P$-channel data are more suitable than the $h$-channel data. The $h$ channel has almost all of its power in the low-frequency band so that events at high frequencies do not contribute enough signal power to trigger a detection. In general, using the $P$-channel for transient detection is equivalent to using the $h$-channel which can be derived from the $P$-channel by linear filtering and therefore contains the same transients.

For the measurement of $P_{c} / Q_{c}$ and $P_{b} / Q_{b}$, whitened data were used as well. The whitening filters for the $P$ - and $Q$-channels were identical so that the results of both the $P_{c} / Q_{c}$ and the $P_{b} / Q_{b}$ measurement are not affected by the whitening process.

The transient detection algorithm was set up with a window length of 45 samples ( $\widehat{=} 2.74 \mathrm{~ms}$ ) each for segments $A$ and $B$ and a length of ten samples ( $\widehat{=} 610 \mu \mathrm{s}$ ) for window $C$ (see figure 2). Measuring the $P_{b} / Q_{b}$ value was done using the same window sizes as for the transient detection. The $P_{c} / Q_{c}$ measurement was carried out with windows $I$ and $I I$ (shown in figure 3 ) set to 20 bins starting 10 bins away from the calibration line frequency. For the vetoing decision, error bars of $\pm 2 \sigma$ were taken around both the $P_{c} / Q_{c}$ and the $P_{b} / Q_{b}$ measurement. This means that a candidate burst event was considered not to be of gravitational wave origin in the case where the intervals $\left[P_{c} / Q_{c}-2 \sigma_{c} ; P_{c} / Q_{c}+2 \sigma_{c}\right]$ and $\left[P_{b} / Q_{b}-2 \sigma_{b} ; P_{b} / Q_{b}+2 \sigma_{b}\right]$ did not overlap.

The transient detection algorithm found an overall number of 24801 events with a SNR greater than 3 during the $\mathrm{S} 1$ run. For 10105 of those events the values of $P_{c} / Q_{c}$ and $P_{b} / Q_{b}$ were so far apart that the event could be vetoed. This is a fraction of $40.7 \%$. 


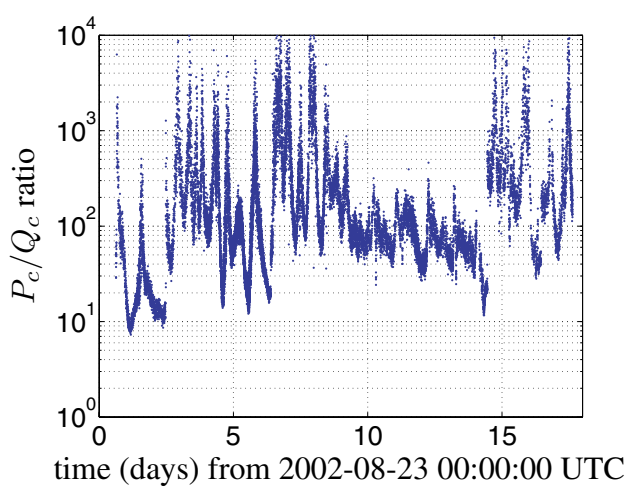

Figure 4. Time evolution of the relative sensitivity $P_{c} / O_{c}$ during the $\mathrm{S} 1$ run.
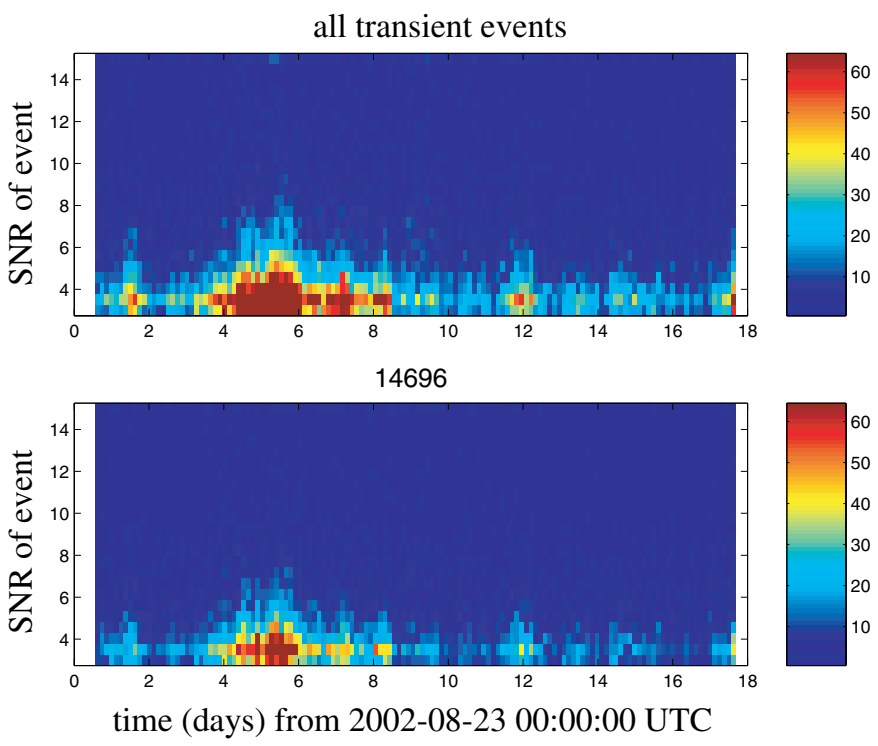

Figure 5. Distribution of transients during the S1 run.

The evolution of the $P_{c} / Q_{c}$ value over time can be seen in figure 4 . The ratio is much greater than 1 most on the time. Glitch events caused by environmental disturbances are expected to act similarly on both the $P$ - and $Q$-channels (e.g. electronic pickup). Therefore, these events should be vetoed efficiently during all the periods where $P_{c} / Q_{c}$ is significantly different from 1.

The upper graph in figure 5 shows the distribution of all detected events broken down into time and SNR bins. The lower graph shows the same plot for the remaining events after the PQMon veto was applied. In figure 6, a histogram of the SNR of the detected transients before and after the vetoing process events is displayed. It can be seen that the efficiency of the vetoing algorithm does not show any particular dependence on the SNR of the transient. The fraction of events vetoed is about $40.7 \%$ using the error bars stated above.

Figures 7 and 8 show two examples of transients that occurred during the S1 run. The left-hand side of figure 7 shows the time series of an event that could be vetoed. On the 

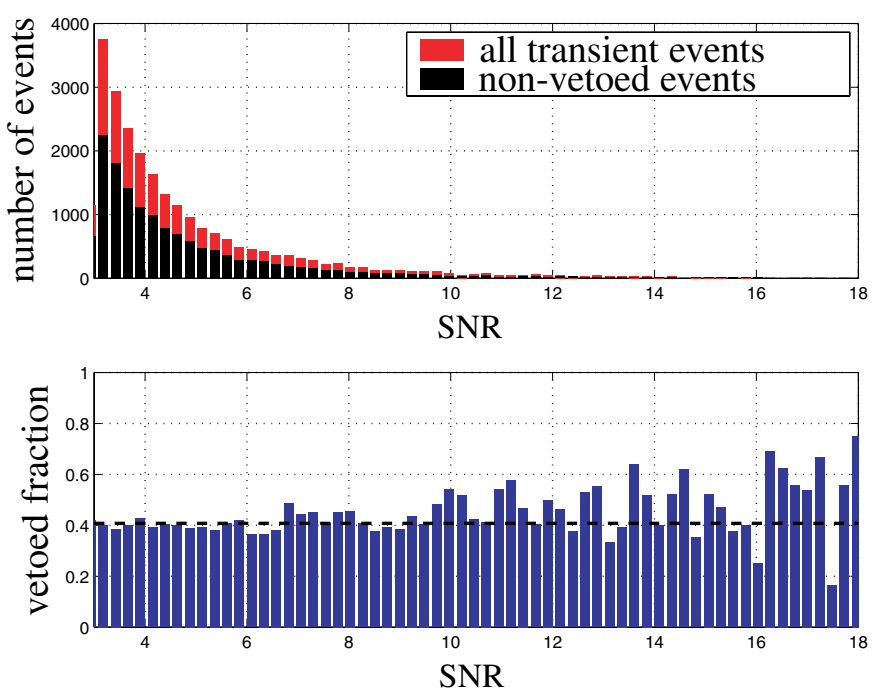

Figure 6. Histogram of candidate burst events before and after the veto process.

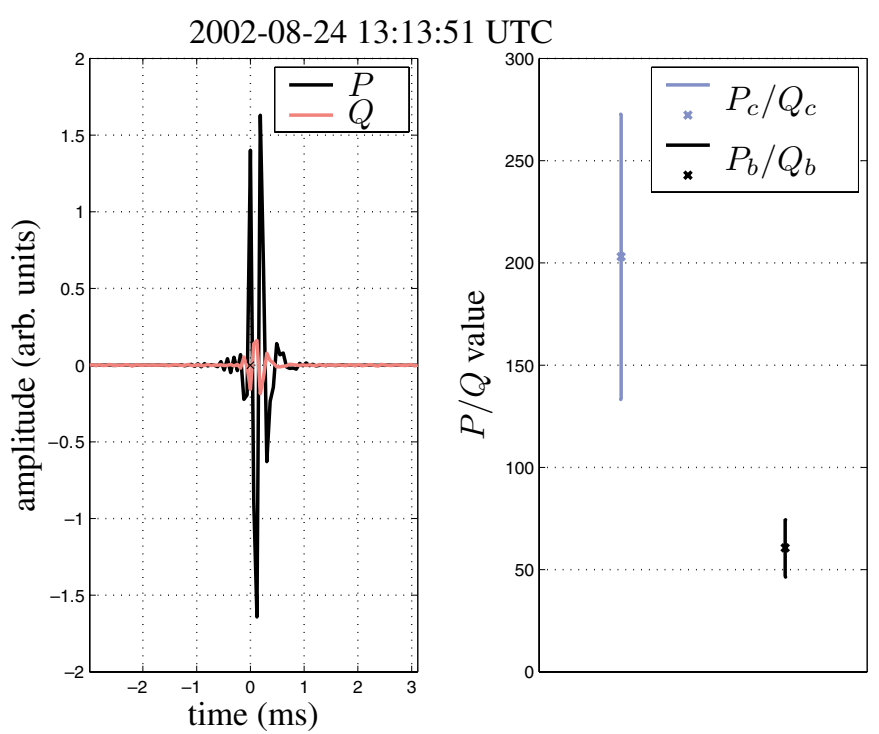

Figure 7. Example of a vetoed event.

right-hand side, the measured values of $P_{c} / Q_{c}$ and $P_{b} / Q_{b}$, including the respective error bars of size $\pm 2 \sigma_{c}$ and $\pm 2 \sigma_{b}$, are displayed: the value of $P_{b} / Q_{b}$ of a gravitational wave induced event predicted by the calibration line measurement is above 200, while the transient showed a $P_{b} / Q_{b}$ value of only about 60 . Looking at the error bars, one can see that this is sufficiently far away from the expected value (error bars do not overlap) for the event to be vetoed.

Figure 8 shows an event that could not be vetoed. The measured value for $P_{b} / Q_{b}$ of about 78 is close enough to the expected value of about 92 for an event induced by a gravitational wave that the error bars of the two measurements overlap. 


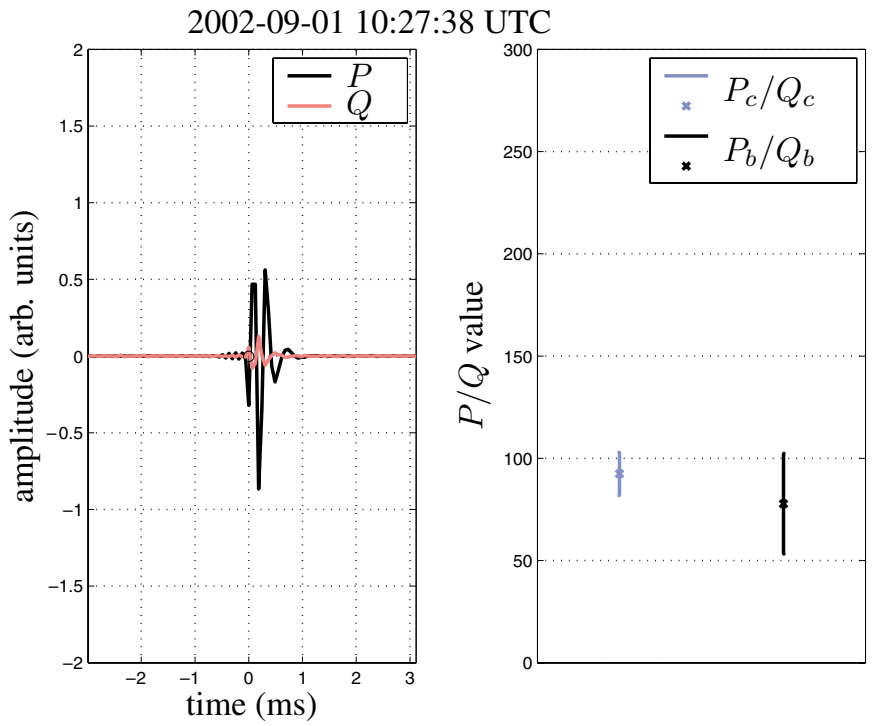

Figure 8. Example of a non-vetoed event.

\section{Outlook}

So far, only the very simple transient detection algorithm explained in section 2.1 is used. It would be desirable to use the vetoing scheme in combination with more powerful algorithms that, for example, break down the signal into several frequency bands.

In addition, the algorithm has to be adapted to work with the future optical configuration of the detector. GEO 600 is in the process of being changed to dual recycling [9] (employing both signal recycling and power recycling). This will make the response to differential arm length changes of the $P$ - and $Q$-channel frequency dependent and therefore require changes of the PQMon algorithm.

\section{References}

[1] Danzmann K et al 1994 GEO 600_Proposal for a 600m Laser-Interferometric Gravitational Wave Antenna (Garching: MPQ)

[2] Webpage http://www.ligo.org/

[3] Abramovici A et al 1992 LIGO - the laser interferometric gravitational-wave observatory Science 256325

[4] Anderson W G, Brady P R, Creighton J D E and Flanagan E E 2001 An excess power statistic for detection of burst sources of gravitational radiation Phys. Rev. D 63042003

[5] Sylvestre J 2002 Time-frequency detection algorithm for gravitational wave bursts Phys. Rev. D 66102004

[6] Hewitson M et al 2003 Proc. 7th Gravitational Waves Data Analysis Workshop (Kyoto, 2002) Class. Quantum Grav. $20 \mathrm{~S} 885$

[7] Webpage http://www.astro.cf.ac.uk/pub/R.Balasubramanian/geo++

[8] LIGO Data Group, VIRGO Data Acquisition Group 2000 Specification of a common data frame format for interferometric gravitational wave detectors LIGO-T970130-C-E, VIRGO-SPE-LAP-5400-102

[9] Heinzel G, Freise A, Grote H, Strain K and Danzmann K 2002 Dual recycling for GEO 600 Class. Quantum Grav. 19 1547-53 